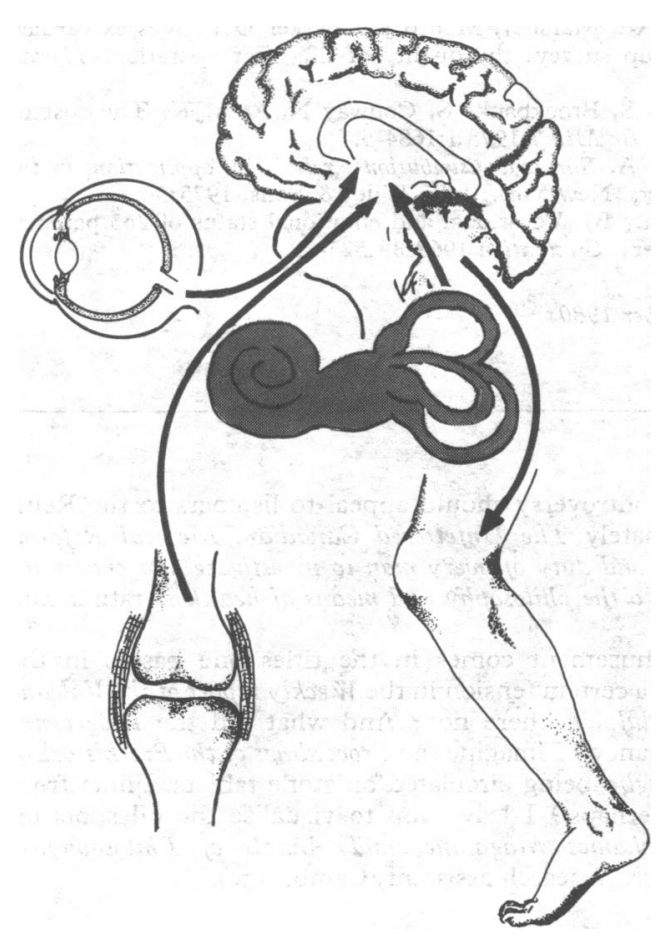

\title{
VERTIGO
}

Vertigo is an illusion of movement, when a patient wrongly believes that he or the environment is moving. It is not a synonym for imbalance; vertigo is always associated with imbalance but imbalance is not always due to vertigo.

Correct balance requires $(a)$ accurate sensory information from the eyes, proprioceptive receptors, and the vestibular labyrinth; (b) accurate co-ordination of this information within the brain; and $(c)$ a functioning motor output from the central nervous system to a normal musculoskeletal system. A defect in any of these systems will impair balance.

Vertigo occurs when the information from vestibular sources conflicts with that from other sensory systems or when a disordered central integration system cannot coherently assess the body's movements from vestibular information. Vertigo is always a symptom of a vestibular defect. The defect may lie in the peripheral labyrinth or in its connections within the brain-the brain stem, the cerebellum, or the temporal lobe cortex. When severe it is usually accompanied by nausea and vomiting.

Vertigo is caused by: (a) peripheral vestibular (labyrinthine) disorders; (b) central vestibular disorders, due to multiple sclerosis, tumours, infarcts, abscesses; and (c) external influences on the vestibular system; external influences include drugs, anaemia, hypoglycaemia, hypotension, viral infection, syphilis, and most important, becuase of its potential danger, erosion of the bony walls of the labyrinth by destructive middle ear disease.

The commonest peripheral vestibular disorders are: Menière's disease and other forms of endolymphatic hydrops; benign paroxysmal positional vertigo; sudden vestibular failure; and vascular disturbances. These account for over $75 \%$ of cases of vertigo.

\section{Menière's disease}

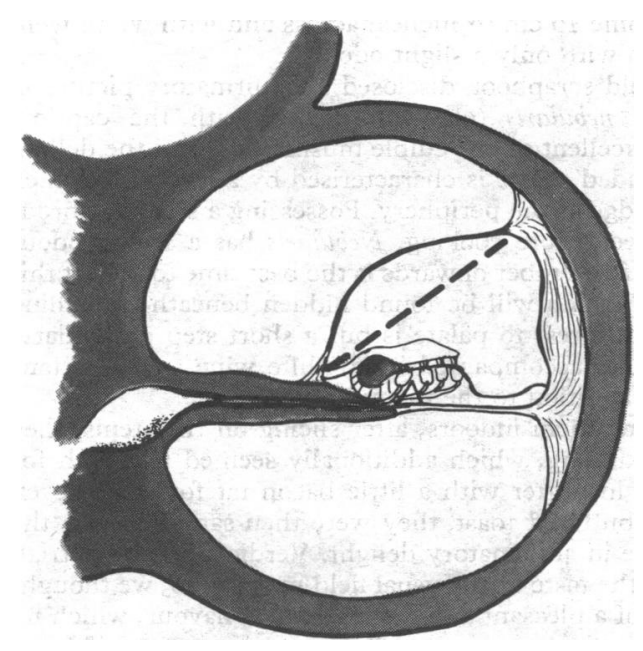

Menière's disease is a disorder of endolymph control characterised by dilatation of the endolymphatic spaces of the membranous labyrinth. Dilatation, or hydrops, may be caused by disorders of the otic capsule such as Paget's disease, labyrinthine syphilis, or previous labyrinthine damage, but in Menière's disease the hydrops is idiopathic.

The disease usually affects only one ear and most often first produces symptoms between the ages of 30 and 60 . It is characterised by attacks of violent paroxysmal vertigo, often rotatory in the direction of disordered sensation, associated with deafness and tinnitus. Attacks usually occur in clusters, with periods of remission in between when balance is normal. An attack lasts for several hours-rarely less than 10 minutes or more than 12 hours-and is generally acompanied by prostration, nausea, and vomiting. A sensation of pressure in the ear, an increase or change in the character of tinnitus, pain in the neck, or increased deafness often precedes an attack. Loss of consciousness is rare and suggests epilepsy.

The accompanying deafness is sensorineural and fluctuates in severity. It is associated with distortion of heard speech and musical sounds and with severe discomfort on exposure to loud noise. The fluctuating hearing loss may develop before the first attack of vertigo, although both symptoms often occur together. Even so, the deafness may not be noticed during the all-consuming experience of vertigo. Hearing tends to improve during 


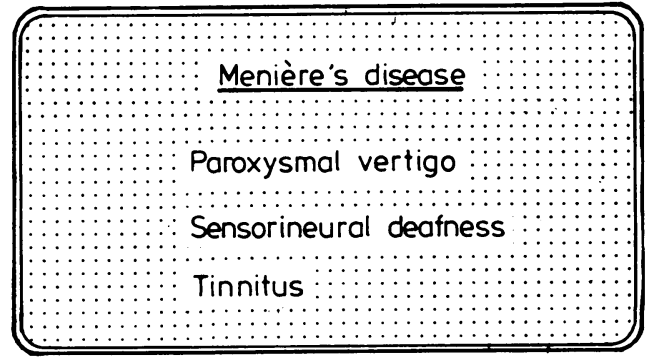

remissions, but over time it deteriorates, fluctuating until the loss becomes severe. The tinnitus is roaring or low pitched and worse when hearing is most impaired.

In the $20-30 \%$ of patients with bilateral Menière's disease deafness tends to become more serious than the vertigo. A form of "vestibular hydrops" produces attacks of Menière-like episodic vertigo, but without auditory symptoms.

\section{Benign paroxysmal (positional) vertigo}

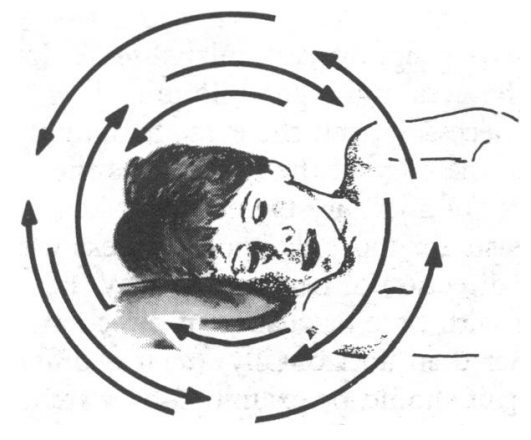

\section{Sudden vestibular failure}

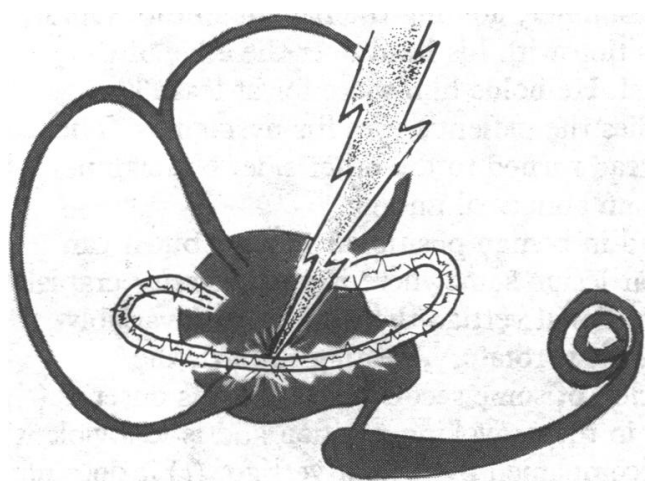

\section{Vascular lesions}

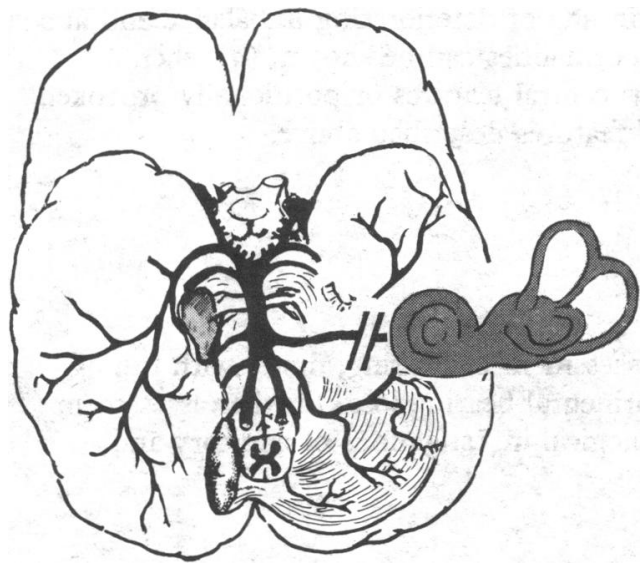

Benign paroxysmal vertigo is provoked by movements of the head, usually to one side when turning in bed or on looking upwards. Each attack lasts for only a few seconds, though it may seem longer to the patient, and there are no auditory symptoms.

The disorder is due to detachment of calcium carbonate crystals from the otoconia of the otolith organ of the affected utricle. These fall against the cupula of the posterior semicircular canal. The cause may be injury, viral infection, or degenerative changes with aging but often there is no explanation. Attacks are provoked only by adopting the specific position of the head and they usually stop after a few weeks or months.

Sudden vestibular failure occurs when one peripheral labyrinth suddenly stops working. This may happen for various reasons, including closed head injuries, viral infection (such as by varicella zoster), blockage of an end artery supplying the labyrinth, multiple sclerosis, diabetic neuropathy, and brain stem encephalitis. The effects are sudden vertigo with prostration, nausea, and vomiting. There are no auditory symptoms, and the vertigo persists continuously, gradually improving over many days or weeks. Vertigo is exacerbated by head movements, but after a few days it may be absent unless the head is moved. The patient gradually regains his balance so that on the third or fourth day after onset he can move unsteadily around the room, holding on to furniture. By the end of 10 days he can usually walk without support if he avoids sudden movement. After three weeks gait may seem normal but the patient still feels insecure, particularly in the dark or when he is tired.

Recovery is slower and less complete in old age. It depends on compensating changes within the brain, and imbalance may return temporarily whenever the acquired compensation breaks down-for example, through defects in other sensory systems, fatigue, other illness, drugs, or the cerebral degeneration of old age.

Migraine may cause episodic vertigo indistinguishable from that of Menière's disease.

Basilar migraine typically affects adolescent girls, causing symptoms similar to those of Menière's disease preceded by dimming or loss of vision due to ischaemia of the posterior cerebral artery territory. The vertigo is often associated with dysarthria and tingling in both hands and feet. Severe occipital headache may follow the attack.

Obstruction of an end artery supplying the labyrinth may produce the syndrome of vestibular failure.

Vertebrobasilar ischaemia is a central vascular disorder. Usually affecting older patients with degenerative vascular disease, it is characterised by attacks of vertigo associated with other symptoms of cerebral ischaemia such as dysphasia, paraesthesiae, weakness of the limbs, and visual disturbance. 


\section{Assessment and testing}

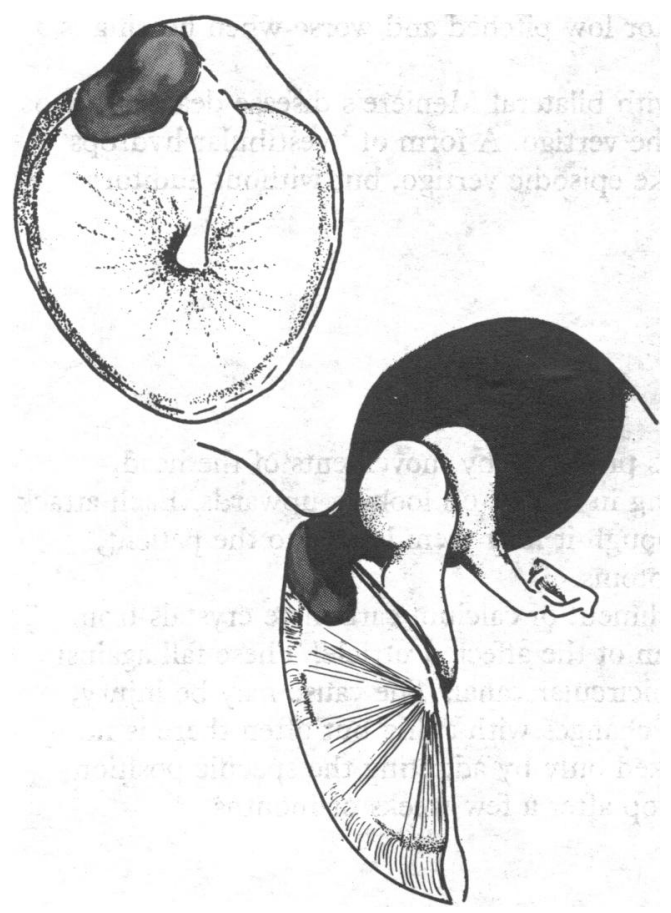

\section{Positional testing}
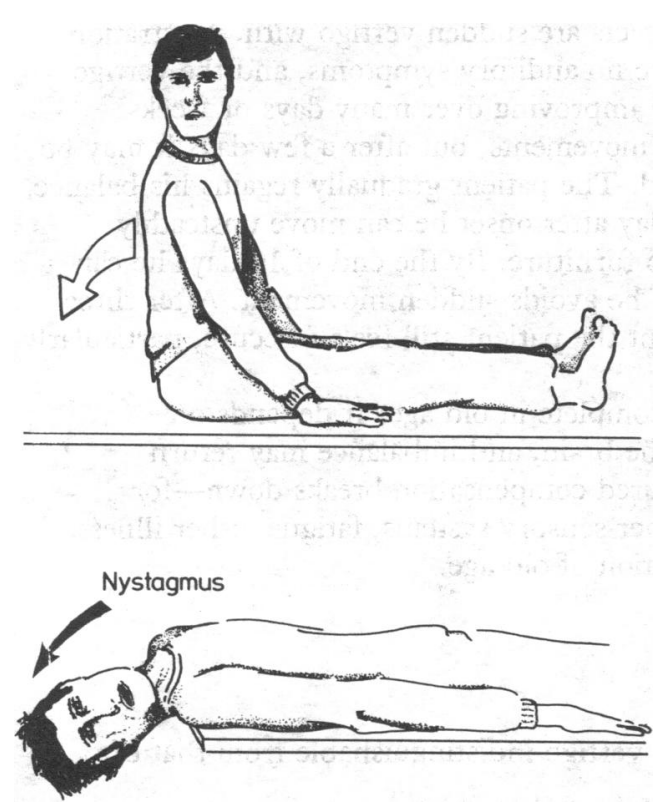

\section{Further tests}

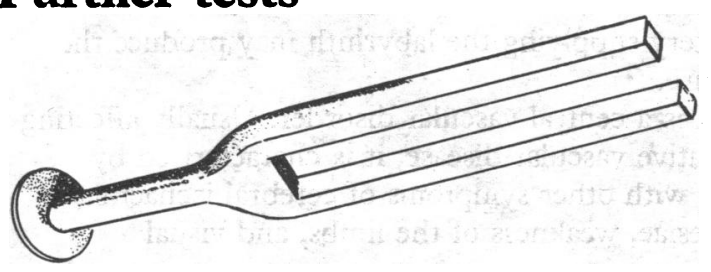

The doctor's first task is to recognise the symptom as vertigo and then to determine whether there is any systemic cause or extralabyrinthine disorder needing urgent investigation-destructive middle ear disease or any central vestibular abnormalities. The history is his most useful tool.

Clinical examination must include assessment of the cardiovascular and central nervous systems. Careful examination of the ears is the only way of recognising destructive middle ear disease with cholesteatoma. Suspicion must remain until each tympanic membrane is found to be normal. A wax crust over the pars flaccida is deceptive since it may cork the entrance to an attic cholesteatoma. If there is any doubt referral for examination under the microscope, which may require general anaesthesia, is essential.

Spontaneous jerk nystagmus is always a sign of vestibular disease. Its assessment requires examination of the eyes under good illumination. Inspection in all positions of gaze is necessary, but the eyes should not be abducted more than about $30^{\circ}$-until the edge of the iris reaches the caruncle. The following characteristics of a jerk nystagmus indicate a central cause: $(a)$ the nystagmus persists for more than a few weeks without abating; $(b)$ it shows a change in the direction of beat (defined by the direction of quick component) either with time or change in direction of gaze; $(c)$ it beats in any direction other than horizontally; $(d)$ it is different in the two eyes (ataxic). Stance and gait should be examined by watching the patient standing with his eyes closed and walking heel to toe.

Simple positional testing is most important. Seated on a couch the patient turns his head towards the examiner and is told to keep his eyes on the examiner's forehead. The examiner, holding the patient's head, rapidly lays him back into a supine position with his head over the edge of the couch $-30^{\circ}$ below the horizontal. He holds him there for at least 30 seconds, during which he watches the patient's eyes for nystagmus. The test is then repeated with the head turned to the other side. Nystagmus provoked by this test is always an abnormal finding.

Positional nystagmus is found in benign positional vertigo, but it can also, rarely, indicate a vestibular lesion somewhere in the posterior cranial fossa. In benign paroxysmal positional vertigo the nystagmus invariably shows the following features: $(a)$ it is rotatory, beating towards the underlying ear; (b) a latent period of some seconds precedes its onset; $(c)$ it abates after 5-20 seconds in the provoking position and is less violent on repeated testing; $(d)$ it is accompanied by violent vertigo; $(e)$ it does not change direction during observation. If any of these features are lacking a central cause must be sought.

Vertigo of central origin should be suspected (a) when there are other neurological symptoms such as visual disturbance, dysphasia, paraethesiae, or weakness of the limbs; $(b)$ when the story does not fit into one of the peripheral labyrinthine patterns, in particular if the vertigo and vomiting are less conspicuous than continuing or deteriorating imbalance and ataxia; (c) when other neurological abnormalities can be shown; $(d)$ when spontaneous jerk nystagmus has central features or positionally provoked nystagmus lacks the peripheral features described above.

Examination next requires assessment of hearing, firstly with tuning fork tests to identify any sensorineural hearing loss. Further assessment demands referral for neuro-otological investigations of auditory and vestibular function. 


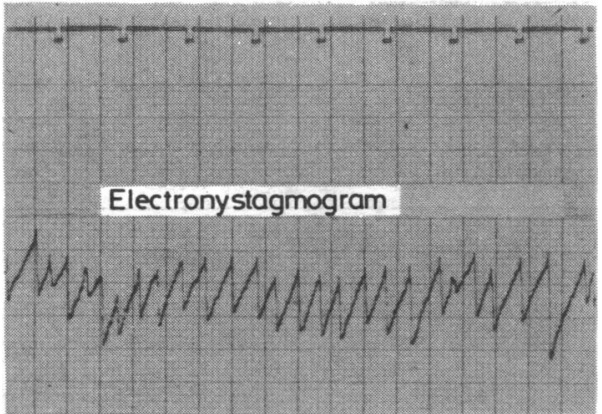

\section{Symptomatic treatment}

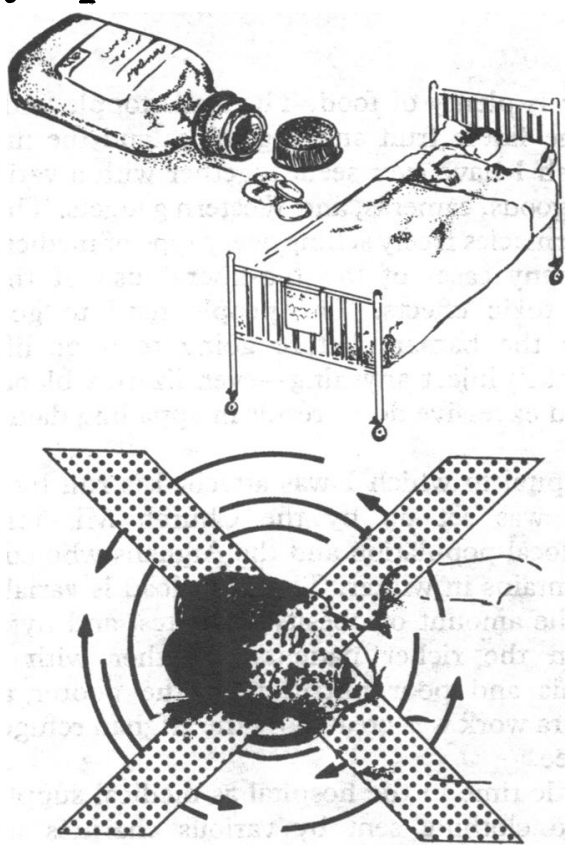

In tests of vestibular function the labyrinth is vicariously stimulated by irrigation of the ears with water at temperatures other than body temperation (caloric tests) or by rotating the whole body at variable angular acceleration. The induced nystagmus may be observed for duration or recorded by electronystagmography (ENG), which allows measurement of such features as the speed of eye movements in a particular direction. ENG also gives valuable diagnostic information about spontaneous nystagmus.

Additional investigations should include serological tests for syphilis.

When conditions demanding referral for further investigation or treatment have been excluded, reassurance to explain that this cataclysmic symptom is not due to serious disease is important. Symptoms may be relieved by sedatives such as prochlorperazine, cinnarizine, and other antihistamines. Diazepam is also useful. If an attack is severe, bedrest will be necessary whatever the cause of the vertigo. If vomiting prevents oral administration of drugs they may be given intramuscularly or as suppositories. Once the acute stage is over sedatives may be continued in small doses for several weeks or months.

If a vestibular deficit, rather than irritation of a more or less normal labyrinthine system, is pronounced vestibular sedatives may exacerbate the symptoms. This often occurs with the degenerative changes of old age; in bilateral Menière's disease and labyrinthine syphilis; or when the labyrinths have been severely damaged by ototoxic drugs. Patients can be helped by graded head and eye movement exercises, designed to accelerate the process of central compensation. These Cawthorne-Cooksey exercises are taught and supervised by physiotherapists. Exercises that cause vertigo may help adaptation in victims of benign positional vertigo, but these patients generally need only to avoid the provoking head position, while those with sudden vestibular failure need reassurance and symptomatic treatment.

\section{Treatment of Menière's disease}

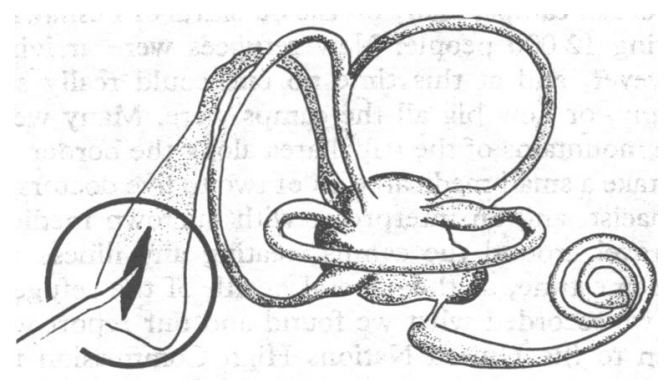

Medical treatment-There is some evidence that the endolymph disturbance has a vascular cause, and vasodilator drugs may be useful; betahistine hydrochloride $8 \mathrm{mg}$ three times a day may be used if there are no contraindications such as a peptic ulcer. Nicotinic acid in a dose sufficient to cause flushing is an alternative. There is also evidence that Menière's disease might be due to electrolyte imbalance, and a saltrestricted diet combined with a diuretic such as frusemide or hydrochlorthiazide may be useful.

Operation may be advised if the symptoms are not controlled by medication and reassurance. Conservative surgical procedures, such as saccus endolymphaticus decompression operations, aim to preserve hearing in the affected ear. These carry little risk of further auditory damage and are valuable in relieving attacks of vertigo; they may also prevent progress of the disease. Other conservative measures include ultrasonic destruction of the vestibulocochlear nerve. Labyrinthectomy, or total destruction of the membranous labyrinth, offers the most certain guarantee of relief from the vertigo of Menière's disease but at the expense of total loss of hearing in that ear. This is acceptable when hearing is already reduced to a distorted shred and the other ear retains normal function.

Other forms of treatment are directed at recognised causes and include surgical exploration of any middle ear in which cholesteatomatous erosion of the middle ear is suspected. If a labyrinthine membrane rupture is suspected after trauma the middle ear may have to be explored to seal a possible perilymph fistula.

Mr Harold Ludman, MA, FRCS, is consultant otolaryngologist, King's College Hospital, and neuro-otological surgeon, National Hospital, Queen Square, London. This is the eighth of 16 articles. 